\section{Polyion Complex (PIC) Flower-shaped Nano-micelles formed from Anionic Triblock and Cationic Diblock Copolymers}

\section{Yuuichi Yokoyama ${ }^{1,2}$, Ryusuke Enomoto ${ }^{1}$ and Shin-ichi Yusa $^{1 *}$}

${ }^{1}$ Department of Materials and Chemistry, University of Hyogo, 2167 Shosha, Himeji, Hyogo, Japan

${ }^{2}$ Taki Chemical Co., Ltd., 64-1 Nishiwaki, Beppucho, Kakogawa, Hyogo, Japan

\begin{abstract}
Poly (sodium 2-(acrylamido)-2-methylpropanesulfonate)-block-poly(ethylene glycol)-block-poly(sodium 2-(acrylamido)-2-methylpropanesulfonate) (PAMPS $\left.48-\mathrm{PEG}_{227}-\mathrm{PAMPS}_{48}\right)$ and poly(ethylene glycol)-block-poly(3-(methacryloylamino)propyl) trimethylammonium chloride PEG $_{47}$-PMAPTAC $m, m=27,53$, and 106) with different PMAPTAC chain lengths were prepared via reversible addition-fragmentation chain transfer controlled living radical polymerization using PEG-based macro chain transfer agents. The subscript numbers in abbreviation represent the degree of polymerization of the block. Mixing of aqueous solutions of the oppositely charged anionic triblock copolymer, $P A M P S_{48}-P E_{227}-$ PAMPS $_{48}$ and cationic diblock copolymers, PEG $_{47}-$ PMAPTAC $_{m}$ led to the spontaneous formation of Polyion Complex (PIC) micelles. The characterization data of NMR, light scattering, $\zeta$-potential, and transmission electron microscopic measurements indicated that the PIC flower micelles were formed comprising the segregated PIC core and surrounded hydrophilic linear and loop PEG shell chains. Aggregation number and hydrodynamic radius achieved maximum values when anionic and cationic block chain lengths of the block copolymers were approximately the same.
\end{abstract}

Keywords: Controlled living radical polymerization; Polyion complex micelle; Water-soluble polymer

${ }^{*}$ Corresponding author: Shin-ichi Yusa, Department of Materials and Chemistry, University of Hyogo, 2167 Shosha, Himeji, Hyogo, Japan, Tel: +81 792674954; Fax: +81 792668868; E-mail: yusa@eng.u-hyogo.ac.jp

Citation: Yokoyama Y, Enomoto R, Yusa S (2014) Polyion Complex (PIC) Flower-shaped Nano-micelles formed from Anionic Triblock and Cationic Diblock Copolymers. J Nanotechnol Nanomed Nanobiotechnol 1: 001.

Received: July 23, 2014; Accepted: October 23, 2014; Published: November 07, 2014

\section{Introduction}

If " $\mathrm{A}$ " and "B" blocks are hydrophilic and hydrophobic blocks, respectively, amphiphilic $\mathrm{AB}$ diblock copolymers form core-shell type polymer micelles in water. The hydrophobic " $\mathrm{B}$ " blocks associate between interpolymer chains to form the hydrophobic core, and the hydrophilic " $\mathrm{A}$ " blocks surround the core as hydrated shells. This kind of core-shell polymer micelles can be applied to a hydrophobic drug carrier, because the drug can be incorporated into the hydrophobic "B" core. In the case of ABA triblock copolymers in water, the polymers form polymer micelles composed of the hydrophobic "B" block core and hydrophilic "A" block shells. Structure of the core-shell polymer micelles formed from $\mathrm{ABA}$ triblock copolymers are similar to that formed from $\mathrm{AB}$ diblock copolymers. On the other hand, in the case of $\mathrm{BAB}$ triblock copolymers in water at the low concentration, the polymers form flower micelles composed of the hydrophobic "B" core and hydrophilic loop "A" shells such as petals [1]. Furthermore, at high concentration of the BAB triblock copolymers, the aqueous solution leads to gelation or precipitation, because network structures are formed due to interpolymer hydrophobic interactions between "B" blocks.

Kadam et al., [2] have reported the synthesis of flower micelles composed of poly (2-methacryloyloxyethyl acrylate)-block-poly(ethylene oxide)-block-poly(2-meth- acryloyloxyethyl acrylate) triblock copolymer bearing polymerizable groups on the hydrophobic blocks. The transient flower micelles structures in water were permanently fixed by cross-linking the methacrylate moieties in the micelles cores under UV light. Graaf et al., [3] have reported that amphiphilic BAB triblock copolymers consisting of Poly (Ethylene Glycol) (PEG) as hydrophilic A block and thermo-responsive poly (N-isopropylacrylamide) (pNIPAm) B blocks from flower micelles above the Lower Critical Solution Temperature (LCST) for pNIPAm blocks in water. These reported examples of flower micelles are based on hydrophobic interactions.

In general, polymer-based nano-aggregates in water are formed due to various driving forces such as interpolymer hydrophobic interactions, hydrogen bonding, Van der Waals, and electrostatic interactions [4-8]. The driving forces of polymer micelle core formation are not only hydrophobic interactions but also electrostatic interactions, which have attracted attention. Kataoka et al., [9-11] reported preparation of oppositely charged double hydrophilic diblock copolymers, poly (ethylene glycol)-block-poly(Llysine) (PEG-P(Lys)) and poly(ethylene glycol)-block-poly( $\alpha, \beta$-asparatic acid) (PEG-P(Asp)). When these oppositely charged diblock copolymers are neutralized in water, water-soluble Polyion Complex (PIC) micelles are formed due to the electrostatic interactions. The PIC micelles are composed of the segregated PIC core formed by charged blocks of cationic P (Lys) and anionic P (Asp), which are surrounded by electrically neutral hydrophilic PEG shells.

We prepared oppositely charged double hydrophilic diblock copolymers (PEG-PMAPTAC and PEG-PAMPS) via Reversible Addition-Fragmentation Chain Transfer (RAFT) controlled living radical polymerization of (3-(Methacryloylamino) Propyl) 
Trimethylammonium Chloride (MAPTAC) and sodium 2-(Acrylamido)-2-Methylpropanesulfonate (AMPS) using PEG-based monofunctional chain transfer agent [12-14]. When these oppositely charged PEG-PMAPTAC and PEG-PAMPS are mixed with stoichiometrically charge neutralization in water, water-soluble PIC micelles are formed, which composed of segregated PIC core composed of cationic PMAPTAC and anionic PAMPS blocks and the outer hydrophilic nonionic PEG shells [15].

In this study, we prepared PIC flower micelles in water (Figure 1). An anionic ABA triblock copolymer (PAMPS ${ }_{48} \mathrm{PEG}_{227}$ PAMPS $_{48}$, AEA) composed of PAMPS and PEG blocks was prepared via RAFT radical polymerization. Cationic diblock copolymers (PEG-PMAPTAC, $\mathrm{EM}_{m}$ ) with different chain lengths of the PMAPTAC block were also prepared via RAFT. When AEA and $\mathrm{EM}_{m}$ were mixed in water, PIC flower micelles were formed through electrostatic interactions between the PAMPS and PMAPTAC blocks (Figure 1). The resulting structures were analyzed using light scattering, NMR, $\zeta$-potential, and Transmission Electron Microscopy (TEM) measurement techniques.

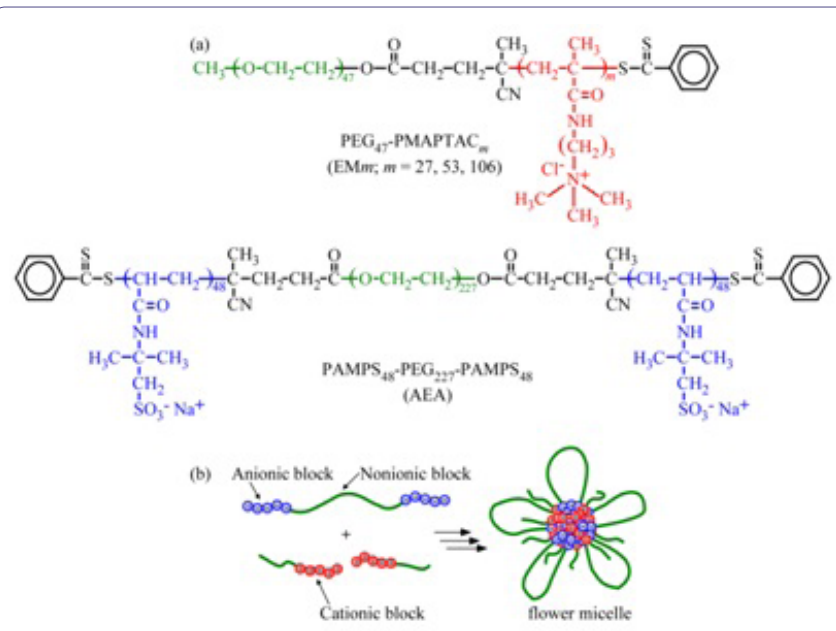

Figure 1: (a) Chemical structures of PAMPS ${ }_{48}-\mathrm{PEG}_{227}-\mathrm{PAMPS}_{48}(\mathrm{AEA})$ and $\mathrm{PEG}_{47}-\mathrm{PMAPTAC}_{m}\left(\mathrm{EM}_{m}, \mathrm{~m}=27,53\right.$, and 106).

(b) Schematic representation of a flower micelle composed of $A E A$ and $E M_{m}$

\section{Materials and Methods}

\section{Chemicals and materials}

4-Cyanopentanoic acid Dithiobenzoate (CPD) was synthesized according to the method reported by McCormick and coworkers [16]. Poly (ethylene glycol)-based chain transfer agent (PEG-CPD, number-average molecular weight $\left(M_{\mathrm{n}}\right)=2.26 \times 103$, number-average Degree of Polymerization $(\mathrm{DP})=47$, molecular weight distribution $\left.\left(M_{\mathrm{w}} / M_{\mathrm{n}}\right)=1.02\right)$ was synthesized as previously reported [15]. (3-(Methacryloylamino)Propyl) Trimethylammonium Chloride (MAPTAC) (50 wt\% in water) from Aldrich was passed through an inhibitor-remover column. $\alpha, \omega$-Bis-hydroxy poly(ethylene glycol) (HO-PEG-OH, number-average molecular weight $\left(M_{\mathrm{n}}\right)=9.40 \times 103$, number-average Degree of Polymerization $(D P)=227$, Molecular weight distribution $\left.\left(M_{\mathrm{w}} / M_{\mathrm{n}}\right)=1.06\right)$ from Aldrich, N,N'-dicyclohexylcarbodiimide (DCC, 99\%) from Kishida Chemical, 4-(N,N-Dimethylamino) Pyridine (DMAP, 99\%), 4,4'-azobis(4-cyanopentanoic acid) (V-501, 98\%), and 2-(Acrylamido)-2-Methylpropanesulfonic acid (AMPS, 95\%) from Wako Pure Chemical were used as received without further purification. Dichloromethane, chloroform, and methanol from Kanto Chemical and Tetrahydrofuran (THF) from Wako Pure Chemical were dried over $4 \AA$ molecular sieves and distilled. Water was purified using a Millipore Milli-Q system. Other reagents were used as received.

\section{Synthesis of poly (ethylene glycol)-based bifunctional chain transfer agent (CPD-PEG-CPD)}

Poly (ethylene glycol)-based bifunctional chain transfer agent (CPD-PEG-CPD) was synthesized according to the literature with slight modifications [17]. A dichloromethane solution $(100 \mathrm{~mL})$ of DCC $(3.15 \mathrm{~g}, 15.3 \mathrm{mmol})$ was added drop wise to a dichloromethane solution $(150 \mathrm{~mL})$ of HO-PEG-OH $\left(M_{\mathrm{n}}=9.40 \times 10^{3}\right.$, $50.1 \mathrm{~g}, 5.01 \mathrm{mmol}), \mathrm{CPD}(3.38 \mathrm{~g}, 12.1 \mathrm{mmol})$, and a trace of DMAP over a period of $30 \mathrm{~min}$. After the reaction mixture was stirred for $20 \mathrm{~h}$ at $40^{\circ} \mathrm{C}$, it was filtrated to remove dicyclohexylurea. The solvent was removed, and the crude product was purified by silica-gel chromatography using a mixture of chloroform and methanol $(9 / 1, v / v)$ as eluent, affording CPD-PEG-CPD as a red powder (42.2 g, 79.0\%). $M_{\mathrm{n}}$ and $M_{\mathrm{w}} / M_{\mathrm{n}}$ were estimated by Gel-Permeation Chromatography (GPC) to be $1.00 \times 104$ and 1.18 , respectively.

\section{Synthesis of PAMPS 48 PEG $_{227}-$ PAMPS $_{48}$ (AEA)}

A predetermined amount of AMPS (3.13 g, $15.1 \mathrm{mmol}$ ) was neutralized with $\mathrm{NaOH}(0.60 \mathrm{~g}, 15.1 \mathrm{mmol})$ in $30.6 \mathrm{~mL}$ of water. To this solution were added predetermined amounts of V-501 (15.5 mg, $0.0552 \mathrm{mmol}$ ). The solution was deoxygenated by purging with Ar gas for $30 \mathrm{~min}$. Polymerization was carried out at $70^{\circ} \mathrm{C}$ for $16 \mathrm{~h}$. After polymerization, the mixture was poured into a large excess of THF to precipitate the resulting polymer which was dialyzed against pure water for one day. The triblock copolymer $\left(\mathrm{PAMPS}_{48}-\mathrm{PEG}_{227}-\mathrm{PAMPS}_{48}\right.$, AEA) was recovered by a freeze-drying technique $(4.05 \mathrm{~g}, 85.8 \%)$. The $M_{\mathrm{n}}$ and $M_{\mathrm{w}} / M_{\mathrm{n}}$ values determined by GPC were $2.32 \times 104$ and 1.42 , respectively. DP for the one PAMPS block was 48 , as estimated by ${ }^{1} \mathrm{H}$ NMR.

\section{Preparation of cationic diblock copolymers (PEG -PMAP- $_{47}$ TAC $\left._{m}\right)[15]$}

A representative example for the preparation of the cationic diblock copolymer is as follows: MAPTAC (5.52 g, $25.0 \mathrm{mmol})$, V-501 (70.1 mg, $0.25 \mathrm{mmol}$ ), and PEG-CPD (1.13 g, $0.50 \mathrm{mmol}$ ) were dissolved in water $(41.0 \mathrm{~mL})$. The mixture was deoxygenated by purging with Ar gas for 30 min. Polymerization was carried out at $70^{\circ} \mathrm{C}$ for $5 \mathrm{~h}$. The polymerization mixture was poured into a large excess of acetone to precipitate the resulting polymer. The polymer was purified by re-precipitating from methanol into a large excess of acetone twice. The cationic diblock copolymer $\left(\mathrm{PEG}_{47}-\mathrm{PMAPTAC}_{53}\right)$ obtained was dried in a vacuum oven at $60^{\circ} \mathrm{C}$ for $24 \mathrm{~h}(5.81 \mathrm{~g}, 87.4 \%)$. The $M_{\mathrm{n}}$ and $M_{\mathrm{w}} / M_{\mathrm{n}}$ values were estimated by GPC to be $1.11 \times 104$ and 1.02 , respectively. DP for the PMAPTAC block was 53 as estimated by ${ }^{1} \mathrm{H}$ NMR.

\section{Preparation of Polyion Complex (PIC) micelles}

AEA and $\mathrm{EM}_{m}$ were separately dissolved in $0.1 \mathrm{M} \mathrm{NaCl}$ aqueous solutions, which were allowed to stand overnight at room temperature to achieve complete dissolution. For the preparation of PIC micelles, an $\mathrm{EM}_{m}$ solution was added drop wise to an AEA solution over a period of $5 \mathrm{~min}$ at room temperature with stirring, and the mixture was allowed to equilibrate for at least one day prior to measurement. The mixing ratio of the two oppositely charged block copolymers was adjusted based on the mole fraction of AMPS units 
$\left(f_{\mathrm{AMPS}}=[\mathrm{AMPS}] /([\mathrm{AMPS}]+[\mathrm{MAPTAC}])\right.$, where $[\mathrm{AMPS}]$ and [MAPTAC] are the mole concentrations of AMPS and MAPTAC units, respectively. The total polymer Concentration $\left(C_{\mathrm{p}}\right)$ for PIC micelles of AEA/EM ${ }_{27}, \mathrm{AEA} / \mathrm{EM}_{53}$, and AEA/EM ${ }_{106}$ were kept constant at $1 \mathrm{~g} / \mathrm{L}$.

\section{Measurements}

GPC measurements for cationic polymer samples were performed using a Shiseido Nanospace SI-1 pump and a Tosoh RI-8012 Refractive Index (RI) detector equipped with a Shodex $10.0 \mu \mathrm{m}$ bead size Ohpak SB-804 HQ column (exclusion limit $\sim 10^{7}$ ) working at $40^{\circ} \mathrm{C}$ with a flow rate of $0.6 \mathrm{~mL} / \mathrm{min}$. A $0.3 \mathrm{M} \mathrm{Na}_{2} \mathrm{SO}_{4}$ aqueous solution containing $0.5 \mathrm{M}$ acetic acid was used as eluent. The values of $M_{\mathrm{n}}$ and $M_{\mathrm{w}} / M_{\mathrm{n}}$ for cationic polymer samples were calibrated using standard poly (2-vinylpyridine) samples of 6 different molecular weights ranging from $5.70 \times 10^{3}$ to $3.16 \times 10^{5}$. GPC measurements for anionic polymer samples were performed using a Tosoh DP-8020 pump and a Tosoh RI-8020 RI detector equipped with a Shodex Asahipak $7.0 \mu \mathrm{m}$ bead size GF-7M HQ column (exclusion limit $\sim 10^{7}$ ) working at $40^{\circ} \mathrm{C}$ with a flow rate of $0.6 \mathrm{~mL} / \mathrm{min}$. A phosphate buffer at $\mathrm{pH} 9$ containing $10 \mathrm{vol} \%$ acetonitrile was used as eluent. The values of $M_{\mathrm{n}}$ and $M_{\mathrm{w}} / M_{\mathrm{n}}$ for anionic polymers were calibrated using standard sodium polystyrene sulfonate samples of 11 different molecular weights ranging from $1.37 \times 10^{3}$ to $2.16 \times 10^{6}$.

${ }^{1} \mathrm{H}$ NMR spectra were obtained using a Bruker DRX-500 spectrometer operating at $500 \mathrm{MHz}$ using deuterium lock at a constant temperature of $20^{\circ} \mathrm{C}$ during the whole run. Sample solutions of the polymer for ${ }^{1} \mathrm{H}$ NMR measurements were prepared in $\mathrm{D}_{2} \mathrm{O}$ containing $0.1 \mathrm{M} \mathrm{NaCl}$ at $C_{\mathrm{p}}=1 \mathrm{~g} / \mathrm{L}$.

Light scattering measurements were performed using an Otsuka Electronics Photal DLS-7000 light scattering equipment with a multi- $\tau$ digital time correlator (ALV-5000/EPP). A He-Ne laser (10.0 $\mathrm{mW}$ at $632.8 \mathrm{~nm}$ ) was used as a light source. Sample solutions for light scattering measurements were filtered using a $0.2 \mu \mathrm{m}$ pore size polytetrafluoroethylene filter. In Static Light Scattering (SLS) measurements, the weight-average molecular weight $\left(M_{\mathrm{w}}\right), z$-average Radius of gyration $\left(\mathrm{R}_{\mathrm{g}}\right)$, and second virial coefficient $\left(\mathrm{A}_{2}\right)$ values were estimated from the relation,

$$
\frac{K C_{\mathrm{p}}}{R_{\theta}}=\frac{1}{M_{\mathrm{w}}}\left(1+\frac{1}{3} R_{\mathrm{g}}^{2} q^{2}\right)+2 A_{2} C_{\mathrm{p}}
$$

where $R_{\theta}$ is the difference between the Rayleigh ratio of the solution and that of the solvent, $K=4 \pi^{2} n^{2}\left(\mathrm{~d} n / \mathrm{d} C_{\mathrm{p}}\right)^{2} / N A \lambda^{4}$ with $\mathrm{d} n /$ $\mathrm{d} C_{\mathrm{p}}$ representing the refractive index increment against $C_{\mathrm{p}}$, NA is Avogadro's number, and $q$ is the magnitude of scattering vector. The $q$ value is calculated from $q=(4 \pi \mathrm{n} / \lambda)(\sin (\theta / 2))$, where $n$ is the refractive index of the solvent, $\lambda$ is the wavelength of light source $(=632.8 \mathrm{~nm})$, and $\theta$ is the scattering angle. The known Rayleigh ratio of toluene was used to calibrate the instrument. Values of $\mathrm{d} n / \mathrm{d} C \mathrm{p}$ at $633 \mathrm{~nm}$ were determined using an Otsuka Electronics Photal DRM-3000 differential refractometer. In the Dynamic Light Scattering (DLS) measurements, to obtain the relaxation time distribution $\tau$ A $(\tau)$, an inverse Laplace Transform (ILT) analysis was performed using the REPES algorithm $[18,19]$. The relaxation rate $(\Gamma=\tau-1)$ is a function of $\theta$ [20]. The Diffusion coefficient $(D)$ is calculated from $D=\left(\Gamma / q^{2}\right)$ $\mathrm{q} \rightarrow 0$. The hydrodynamic radius $\left(R_{\mathrm{h}}\right)$ is given by the Stokes-Einstein equation, $R_{\mathrm{h}}=k_{\mathrm{B}} T /(6 \pi \eta \mathrm{D})$, where $k_{\mathrm{B}}$ is the Boltzmann constant, $T$ is the absolute temperature, and $\eta$ is the solvent viscosity. The details of DLS instrumentation and theory are described in the literature [21]. $\zeta$-potential measurements were performed using a Malvern Zetasizer Nano-ZS equipped with a He-Ne laser light source ( $4 \mathrm{~mW}$ at $632.8 \mathrm{~nm}$ ) at $20^{\circ} \mathrm{C}$. $\zeta$-potential was calculated from the electrophoretic mobility $(\mu)$ using the Smoluchowski relationship, $\zeta=\eta \mu / \varepsilon$ (ка >> 1) where $\eta$ is the viscosity, $\varepsilon$ is the dielectric constant of the medium, and $\kappa$ and $a$ are the Debye-Hückel parameter and the particle radius, respectively [22].

Transmission Electron Microscopy (TEM) observations were carried out with a JEOL JEM-2100 microscope at an accelerating voltage of $200 \mathrm{kV}$. Samples for TEM observations were prepared by placing one drop of aqueous solution on a copper grid coated with thin films of Formvar. Excess water was blotted using a filter paper. The samples were stained with sodium phosphotungstate and dried under vacuum for one day.

\section{Results and Discussion}

$\mathrm{PEG}_{47}$-PMAPTAC $m$ was prepared via RAFT of cationic MAPTAC using PEG-CPD in our laboratory previously, which was used in this study [15]. In Figure 2, a time-conversion relationship is depicted along with the first-order kinetic plot for polymerization of AMPS. There was an induction period of $238 \mathrm{~min}$, which may be due to the slow rate of the formation of the 4-cyanopentanoic acid radical fragment, as reported by McCormic and coworkers [23]. The kinetic plot for RAFT polymerization of AMPS, shown in Figure 2, indicates that the concentration of the propagating radical remained constant during the polymerization.

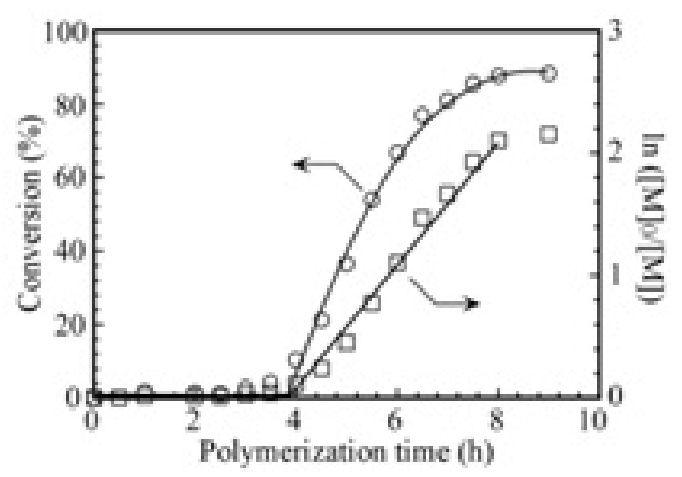

Figure 2: Time-conversion ( $\circ$ ) and the first-order kinetic plots ( $\square$ ) for the polymerization of AMPS in the presence of CPD-PEG-CPD in water at $70^{\circ} \mathrm{C}$. $[\mathrm{M}]_{0}$ and $[\mathrm{M}]$ represent the concentrations of the monomer at polymerization time $=0$ and the corresponding time, respectively.

Figure 3 compares GPC elution curves (RI response) for HO-PEG$\mathrm{OH}$ and $\mathrm{PAMPS}_{48}-\mathrm{PEG}_{227}-\mathrm{PAMPS}_{48}$. Values of $M_{\mathrm{n}}$ and $M_{\mathrm{w}} / M_{\mathrm{n}}$ for all the block copolymers are listed in Table 1. $\mathrm{PAMPS}_{48}-\mathrm{PEG}_{227}{ }^{\mathrm{n}} \mathrm{PAMPS}_{48}$ and $\mathrm{PEG}_{47}-\mathrm{PMAPTAC}_{m}$, are further abbreviated as AEA and $\mathrm{EM}_{m}$, A, E, M, and $m$ representing PAMPS, PEG, PMAPTAC, and DP of PMAPTAC, respectively.

Figure 4 shows ${ }^{1} \mathrm{H}$ NMR spectra of $\mathrm{EM}_{53}, \mathrm{AEA}$, and AEA/ $\mathrm{EM}_{53}$ micelle in $\mathrm{D}_{2} \mathrm{O}$ containing $0.1 \mathrm{M} \mathrm{NaCl}$. DP $(=m)$ and $M_{\mathrm{n}}(\mathrm{NMR})$ of the PMAPTAC block in $\mathrm{EM}_{m}$ were determined from the integral intensity ratio of the resonance bands due to the pendant methyl and methylene protons in the PMAPTAC block around 3.1 to $3.4 \mathrm{ppm}$ and the PEG main chain protons at $3.8 \mathrm{ppm}$. DP and $M_{\mathrm{n}}$ (NMR) of the PAMPS block in AEA were calculated from the integral intensity ratio of the resonance bands due to the pendent methylene protons in the PAMPS block at $3.4 \mathrm{ppm}$ and PEG main chain protons at $3.8 \mathrm{ppm}$. Figure $4 \mathrm{c}$ shows the ${ }^{1} \mathrm{H}$ NMR spectrum of a stoichiometrically charge 


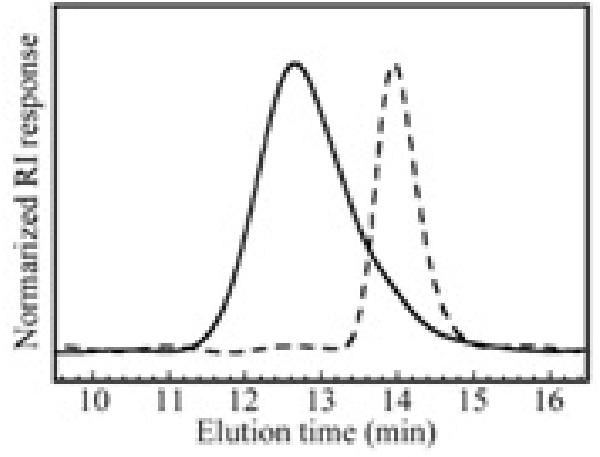

Figure 3: GPC elution curves for a sample of HO-PEG-OH $\left(\mathrm{M}_{n}=9.40 \times 103\right.$; $\left.\mathrm{M}_{\mathrm{w}} / \mathrm{M}_{\mathrm{n}}=1.06\right)$ (----) and triblock copolymer of $\mathrm{PAMPS}_{48}-\mathrm{PEG}_{227}-\mathrm{PAMPS}_{48}$ $\left(A E A, M_{n}=2.32 \times 104 ; M_{w} / M_{n}=1.42\right)(-)$.

neutralized mixture of $\mathrm{EM}_{53}$ and $\mathrm{AEA}$ in $\mathrm{D}_{2} \mathrm{O}$ containing $0.1 \mathrm{M} \mathrm{NaCl}$. The intensities of the resonance bands associated with the PMAPTAC pendent methyl protons at $3.1 \mathrm{ppm}$ and PAMPS pendent methyl protons at $1.5 \mathrm{ppm}$ were extremely weak compared with those associated with the PEG main chain protons at $3.8 \mathrm{ppm}$. This observation suggested that motions of the PMAPTAC and PAMPS blocks were restricted as a result of the formation of PIC by these oppositely charged block chains. On the other hand, the motion of the PEG blocks was not restricted due to the formation of PEG shells.

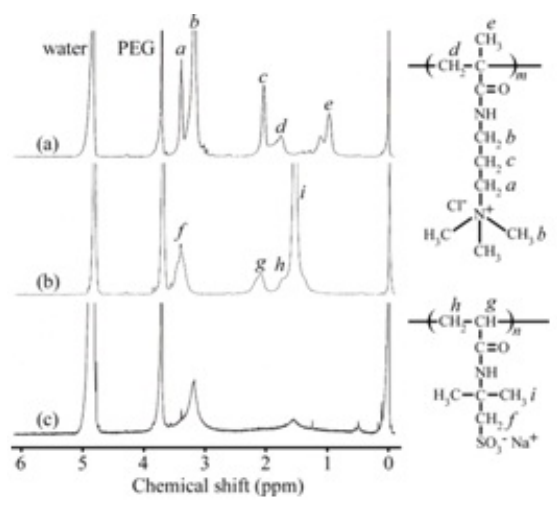

Figure 4: ${ }^{1} \mathrm{H}$ NMR spectra for (a) $\mathrm{EM}_{53}$, (b) AEA, and (c) AEA/EM ${ }_{53}$ micelle in $\mathrm{D}_{2} \mathrm{O}$ containing $0.1 \mathrm{M} \mathrm{NaCl}$ at $20^{\circ} \mathrm{C}$. Assignments are indicated for the resonance peaks.

If the polymerization is assumed to be ideally living in nature, then the theoretical $M_{\mathrm{n}}\left(M_{\mathrm{n}}(\right.$ theo $\left.)\right)$ can be calculated as

$$
M_{\mathrm{n}}(\text { theo })=\frac{[\mathrm{M}]_{0}}{[\mathrm{CTA}]_{0}} \frac{x_{\mathrm{m}}}{100} M_{\mathrm{m}}+M_{\text {СTA }}
$$

where $[\mathrm{M}]_{0}$ is the initial molar concentration of monomer, $[\mathrm{CTA}]_{0}$ is the initial molar concentration of Chain Transfer Agent (CTA), $x_{\mathrm{m}}$ is the percentage conversion of the monomer, $M_{\mathrm{m}}$ is the molecular weight of the monomer, and MCTA is the molecular weight of CTA. The $M_{\mathrm{n}}(\mathrm{NMR})$ values for $\mathrm{EM}_{m}$ and AEA were calculated from ${ }^{1} \mathrm{H}$ NMR data. As shown in Table 1, the $M_{\mathrm{n}}(\mathrm{NMR})$ values for $\mathrm{EM}_{m}$ and AEA were in reasonable agreement with $M_{\mathrm{n}}$ (theo). However, the $M_{\mathrm{n}}$ (theo) and $M_{\mathrm{n}}(\mathrm{GPC})$ values for $\mathrm{EM}_{m}$ and AEA were slightly different, because poly(2-vinylpyridine) or poly(sodium styrenesulfonate) were used as a standard polymer to calibrate $M_{\mathrm{n}}(\mathrm{GPC})$, respectively, and its volume-to-mass ratio may be different from that of EM $m$ and AEA [24].

\begin{tabular}{|c|c|c|c|c|c|c|}
\hline Samples & $\begin{array}{c}\mathbf{M}_{\mathbf{n}}(\mathbf{t h e o}) \\
\mathbf{a} \times \mathbf{1 0}^{-4}\end{array}$ & $\begin{array}{c}\mathbf{M}_{\mathbf{n}}(\mathbf{N M R})^{\mathbf{b}} \\
\times \mathbf{x 1 0}^{-4}\end{array}$ & $\begin{array}{c}\mathbf{M}_{\mathbf{n}}(\mathbf{G P C})^{\mathbf{c}} \\
\mathbf{\times 1 0 ^ { - 4 }}\end{array}$ & $\mathbf{M}_{\mathbf{w}} / \mathbf{M}_{\mathbf{n}}{ }^{\mathbf{c}}$ & $\mathbf{R}_{\mathbf{h}}{ }^{\mathbf{d}}(\mathbf{n m})$ & $\begin{array}{c}\zeta \text {-potential } \\
(\mathbf{m V} \mathbf{)}\end{array}$ \\
\hline $\mathrm{EM}_{27}$ & 0.78 & 0.83 & 0.82 & 1.03 & 4.5 & 18.2 \\
\hline $\mathrm{EM}_{53}$ & 1.36 & 1.41 & 1.11 & 1.02 & 4.3 & 24.2 \\
\hline $\mathrm{EM}_{106}$ & 2.52 & 2.58 & 1.51 & 1.02 & 6.1 & 25.4 \\
\hline $\mathrm{AEA}$ & 3.21 & 3.26 & 2.32 & 1.42 & 6.1 & -14.4 \\
\hline
\end{tabular}

Table 1: Number-average Molecular weight $\left(\mathrm{M}_{\mathrm{n}}\right)$, Molecular weight distribution $\left(M_{w} / M_{n}\right)$, hydrodynamic radius $\left(R_{h}\right)$, and $\zeta$-potential for the polymers.

${ }^{\mathrm{a} C}$ Calculated from Equation (2), ${ }^{\mathrm{b}}$ Estimated from ${ }^{1} \mathrm{H}$ NMR, ${ }^{\mathrm{C}}$ Estimated from GPC, dEstimated from DLS.
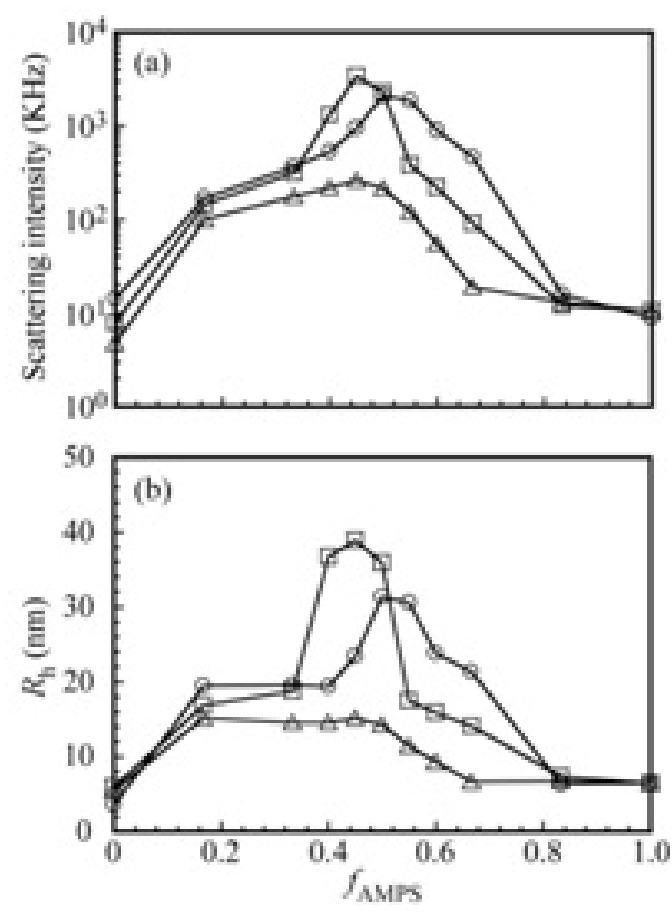

Figure 5: (a) Light scattering intensities and (b) $R_{h}$ for PIC micelles of

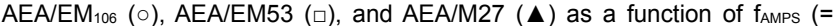
[AMPS]/([AMPS] + [MAPTAC])) in $0.1 \mathrm{M} \mathrm{NaCl}$ aqueous solutions. [AMPS] and [MAPTAC] represent the concentrations of the AMPS and MAPTAC units, respectively. The total polymer concentration was kept constant at $1 \mathrm{~g} / \mathrm{L}$.

Figure 4 shows light scattering intensities and hydrodynamic radius $\left(R_{\mathrm{h}}\right)$ for a mixture of AEA and $\mathrm{EM}_{m}$ in $0.1 \mathrm{M} \mathrm{NaCl}$ as a function of $f_{\text {AMPS }}(=[$ AMPS $] /([$ AMPS $]+[$ MAPTAC $]))$. The total polymer concentration $\left(C_{\mathrm{p}}\right)$ was kept constant at $1 \mathrm{~g} / \mathrm{L}$. At $C_{\mathrm{p}}=1 \mathrm{~g} / \mathrm{L}$ of $\mathrm{AEA} / \mathrm{EM}_{m}$, an increase in viscosity of the solution cannot be observed, which indicates that network formation due to open association of interpolymer electrostatic interaction cannot be occurred. If the $C_{\mathrm{p}}$ value increases, the solution viscosity may increase. However, in this study we focused on PIC flower micelles at diluted state $\left(C_{\mathrm{p}} \leq 1 \mathrm{~g} / \mathrm{L}\right)$. An increase in the scattering intensity indicates an increase in the size of the micelle. Maximum $R_{\mathrm{h}}$ and scattering intensity were observed at close to stoichiometric charge neutralization of PAMPS and PMAPTAC segments. The PIC micelles with maximum $R_{\mathrm{h}}$ and scattering intensity, i.e., $\mathrm{AEA} / \mathrm{EM}_{106}$, $\mathrm{AEA} / \mathrm{EM}_{53}$, and $\mathrm{AEA} / \mathrm{EM}_{27}$ with $f_{\mathrm{AMPS}}=45,50$, and $45 \%$, respectively, were used in this study unless otherwise stated.

$\zeta$-potential of $\mathrm{EM}_{m}$ and AEA are presented in Table 1 . The $\zeta$-potential values for $\mathrm{EM}_{m}$ were positive values, which increased with 
increasing DP of the cationic PMAPTAC block. The $\zeta$-potential value for AEA was negative due to pendant sulfonate anions in the PAMPS blocks. When $\mathrm{EM}_{m}$ and AEA were mixed to prepare PIC micelles, the $\zeta$-potential values for PIC micelle of AEA/EM ${ }_{m}$ were close to zero (Table 2). This observation suggested that AEA and $\mathrm{EM}_{m}$ were almost stoichiometric charge neutralization of PAMPS and PMAPTAC segments.

\begin{tabular}{|c|c|c|c|c|c|c|c|}
\hline \multirow{2}{*}{ PIC micelles } & \multirow{2}{*}{$M_{w^{\mathrm{a}}} \times 10^{-5}$} & $R_{\mathrm{g}}{ }^{a}$ & $R_{\mathrm{h}^{\mathrm{b}}}$ & \multirow{2}{*}{$R_{\mathrm{g}} / R_{\mathrm{h}}$} & \multirow{2}{*}{$N_{\text {agg }}{ }^{\circ}$} & \multirow{2}{*}{$d^{P_{P I C}}{ }^{d}$} & \multirow{2}{*}{$\begin{array}{l}\zeta \text {-potential } \\
(\mathrm{mV})\end{array}$} \\
\hline & & $(\mathrm{nm})$ & $(\mathrm{nm})$ & & & & \\
\hline AEA/EM 27 & 8.48 & 15.1 & 15.2 & 0.99 & 50 & 0.096 & -0.88 \\
\hline AEA/EMs3 & 189 & 36.6 & 41.0 & 0.89 & 735 & 0.109 & -0.53 \\
\hline AEA/EM106 & 111 & 28.6 & 32.4 & 0.88 & 302 & 0.129 & -0.20 \\
\hline
\end{tabular}

Table 2: Dynamic and static light scattering data for PIC micelles in $0.1 \mathrm{M} \mathrm{NaCl}$.

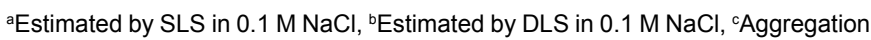
number of PIC micelles calculated from $\mathrm{M}_{\mathrm{w}}(\mathrm{SLS})$ of PIC micelles determined by SLS and $\mathrm{M}_{w}$ of the corresponding unimers determined by ${ }^{1} \mathrm{H}$ NR and GPC ${ }^{\mathrm{d} D e n s i t y ~ c a l c u l a t e d ~ f r o m ~ E q u a t i o n ~(3) . ~}$

The $R_{\mathrm{h}}$ and scattering intensity values of $\mathrm{AEA} / \mathrm{EM}_{53}$ micelle were the largest compared to those of $\mathrm{AEA} / \mathrm{EM}_{106}$ and $\mathrm{AEA} / \mathrm{EM}_{27}$ micelles. Kataoka et al., reported that when the anionic and cationic chain length of block copolymers are approximately the same, the aggregation number $\left(N_{\mathrm{agg}}\right)$, defined as the total number of polymer chains forming one micelle, is the largest of the PIC micelles [11]. The values of DP for anionic PAMPS in AEA and cationic PMAPTAC in $\mathrm{EM}_{53}$ are 48 and 53, respectively. AEA and $\mathrm{EM}_{53}$ may form complex easily, because these DP values of charged blocks in $\mathrm{AEA} / \mathrm{EM}_{53}$ are closer than those of $\mathrm{AEA} / \mathrm{EM}_{106}$ and $\mathrm{AEA} / \mathrm{M}_{27}$. Therefore, a pair of AEA/EM $\mathrm{E}_{53}$ micelles may have the largest $N_{\mathrm{agg}}$.

Values of $R_{\mathrm{h}}$ for the block copolymers were determined by DLS at $C_{\mathrm{p}}=1 \mathrm{~g} / \mathrm{L}$ in $0.1 \mathrm{M} \mathrm{NaCl}$, as listed in Table 1 . The $R_{\mathrm{h}}$ values ranging from 4.5 to $6.1 \mathrm{~nm}$ appear to be reasonable for unimers of these block copolymers. Figure 6 a shows $R_{\mathrm{h}}$ distributions for AEA/EM $\mathrm{E}_{m}$ micelles. The values of $R_{\mathrm{h}}$ estimated from the distributions were summarized in Table 2. The $R_{\mathrm{h}}$ values of AEA/EM $\mathrm{EM}_{106}, \mathrm{AEA} / \mathrm{EM}_{53}$, and AEA/EM 27 micelles were $32.4,41.0$, and $15.2 \mathrm{~nm}$, respectively. When $1.5 \mathrm{M} \mathrm{NaCl}$ was added to the $\mathrm{AEA} / \mathrm{EM}_{53}$ aqueous solution, the $R_{\mathrm{h}}$ value decreased. This observation suggests that the micelle is dissociated by adding $\mathrm{NaCl}$.

The relaxation rates $(\Gamma)$ measured at different scattering angles $(\boldsymbol{\theta})$ were plotted as a function of the square of the magnitude of the scattering vector $\left(q^{2}\right)$ in Figure $6 \mathrm{~b}$. A linear relation passing through the origin indicates that the relaxation modes are virtually diffusive [25]. The $R_{\mathrm{b}}$ value estimated from slope of the $\Gamma$ versus $q^{2}$ plot, was found to be in good agreement with the $R_{\mathrm{h}}$ value calculated from the peak of the $R_{\mathrm{h}}$ distribution obtained at $\theta=90^{\circ}$ (Figure 6a). Because the angular dependence was negligible, $R_{\mathrm{b}}$ values were estimated at a fixed $\theta$ of $90^{\circ}$. In Figure $6 \mathrm{c}$, the $R_{\mathrm{h}}$ values are plotted against $C_{\mathrm{p}}$. The $R_{\mathrm{h}}$ values of $\mathrm{AEA} / \mathrm{EM}_{106}, \mathrm{AEA} / \mathrm{EM}_{53}$, and $\mathrm{AEA} / \mathrm{EM}_{27}$ micelles were approximately 32,41 , and $15 \mathrm{~nm}$, respectively, which were practically constant independent of $C_{\mathrm{p}}$ in the range of 0.2 to $1 \mathrm{~g} / \mathrm{L}$. From DLS results, the stoichiometrically charge neutralized mixture of AEA and $\mathrm{EM}_{m}$ may form flower micelles without intermicellar aggregates because of the unimodal $R_{\mathrm{h}}$ distributions and independence of $C_{\mathrm{p}}$ in the range of 0.2 to $1 \mathrm{~g} / \mathrm{L}$.

Figure 7 shows a typical example of Zimm plots for AEA/EM micelle. Apparent values of $M_{\mathrm{w}}$ and $R_{\mathrm{g}}$, determined from Zimm plots, were listed in Table 2. $N_{\text {agg }}$ can be calculated from the ratio of $M_{\mathrm{w}}$ values for PIC micelle and unimer. $N_{\mathrm{agg}}$ for $\mathrm{AEA} / \mathrm{EM}_{27}, \mathrm{AEA} / \mathrm{EM}_{53}$, and
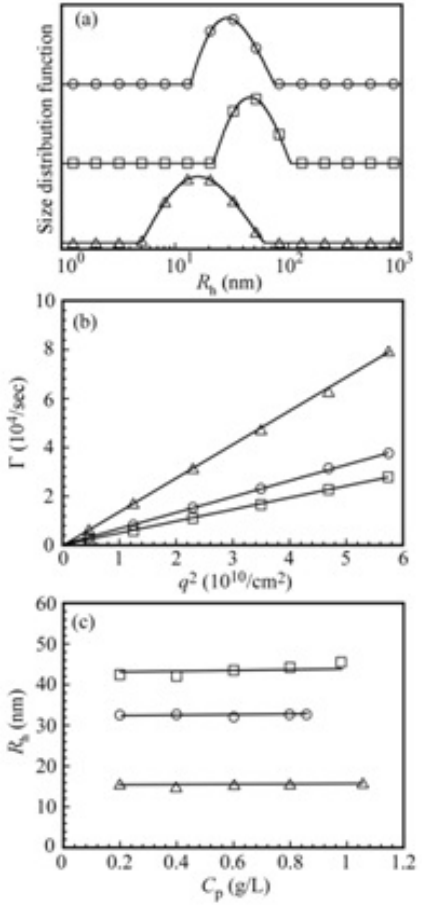

Figure 6: (a) Distributions of $R_{h}$ for the PIC micelles of $A E A / E M_{106}(0)$, $A E A / E M_{53}$ (口), and $A E A / E M_{27}(\boldsymbol{\Delta})$ in $0.1 \mathrm{M} \mathrm{NaCl}$ aqueous solutions. (b) Relationship between relaxation rate $(\Gamma)$ and square of the magnitude of the scattering vector $\left(q^{2}\right)$. (c) Plots of $R_{h}$ as a function of $C_{p}$.

AEA/EM ${ }_{106}$ micelles were 50,735 and 302 , respectively. $N_{\text {agg }}$ for AEA/ $\mathrm{EM}_{53}$ micelle shows maximum number compared with those of AEA/ $\mathrm{EM}_{27}$ and AEA/EM106 micelles.

The $R_{\mathrm{g}} / R_{\mathrm{h}}$ value is useful for characterizing the shape of molecular assemblies. The theoretical value of $R_{g} / R_{\mathrm{h}}$ for a homogeneous hard sphere is 0.778 , however the ratio increases substantially for less dense structures and polydisperse mixtures; for example, $R_{\mathrm{g}} / R_{\mathrm{h}}=1.5$ to 1.7 for flexible linear chains in good solvents, whereas $R_{\mathrm{g}} / R_{\mathrm{h}} \geq 2$ for a rigid rod [26-28]. As shown in Table 2, the $R_{\mathrm{g}} / R_{\mathrm{h}}$ ratios for the micelle were found to be $0.88-0.99$, which suggested that the shape of PIC micelles was fairly close to spherical shape.

The density of PIC micelles ( $\left.d_{\text {PIC }}\right)$ can be calculated by

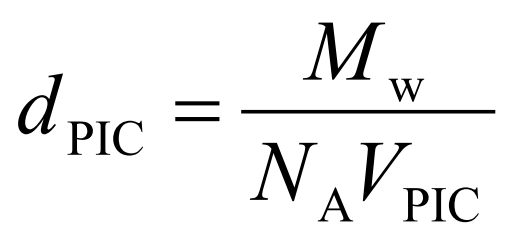

where NA is Avogadro's number and $V_{\mathrm{PIC}}$ is the volume of a PIC micelle. $V_{\text {PIC }}$ can be calculated to be $V_{\text {PIC }}=4 \pi R_{\mathrm{h}}{ }^{3} / 3$. Values of $d_{\text {PIC }}$ for $\mathrm{AEA} / \mathrm{EM}_{27}, \mathrm{AEA} / \mathrm{EM}_{53}$ and AEA/EM ${ }_{106}$ micelles were calculated to be $0.096,0.109$ and $0.129 \mathrm{~g} / \mathrm{cm}^{3}$, respectively. These values are close to the density $\left(d_{\text {PIC }}=0.050-0.148 \mathrm{~g} / \mathrm{cm}^{3}\right)$ of PIC micelles formed from the mixture of PEG-P (Lys) with PEG-P(Asp) [9]. The $d_{\text {PIC }}$ value for $\mathrm{AEA} / \mathrm{EM}_{106}$ micelle with long cationic PMAPTAC block was larger than that for AEA/EM 27 micelle with short cationic PMAPTAC block. This observation suggested that PIC micelle of AEA/EM $m$ with short cationic block length may be more hydrated, i.e., the content of water 
molecules in AEA/EM 27 micelle may be larger than that in AEA/EM micelle, because the volume of PEG chains in one AEA/EM micelle $_{27}$ was larger than those in $\mathrm{AEA} / \mathrm{EM}_{53}$ and $\mathrm{AEA} / \mathrm{EM}_{106}$ micelles.

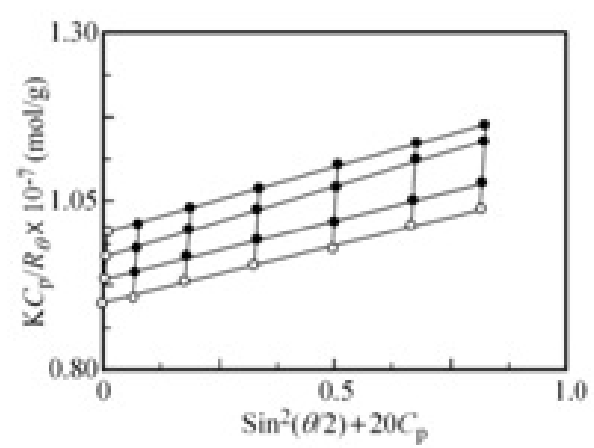

Figure 7: A typical example of Zimm plots for $A E A / E M_{106}$ micelle in $0.1 \mathrm{M} \mathrm{NaCl}$ aqueous solution.

Figure 8 shows TEM images for PIC micelles composed of $\mathrm{AEA} / \mathrm{EM}_{27}, \mathrm{AEA} / \mathrm{EM}_{53}$, and AEA/EM ${ }_{106 .}$ The average diameters of PIC flower micelles composed of AEA/EM ${ }_{27}, \mathrm{AEA}_{\mathrm{EM}}$ and AEA/EM estimated from TEM were $14 \pm 4,53 \pm 2$ and $49 \pm 5 \mathrm{~nm}$, respectively, which were smaller than the $2 R_{\mathrm{h}}$ values, estimated from DLS (Table 2). This implied that the PIC micelles shrank after the removal of water in dry state to measure TEM [29]. Spherical shape for PIC micelles can be observed, suggesting that the aggregates are individual flower micelles without intermicellar aggregation.

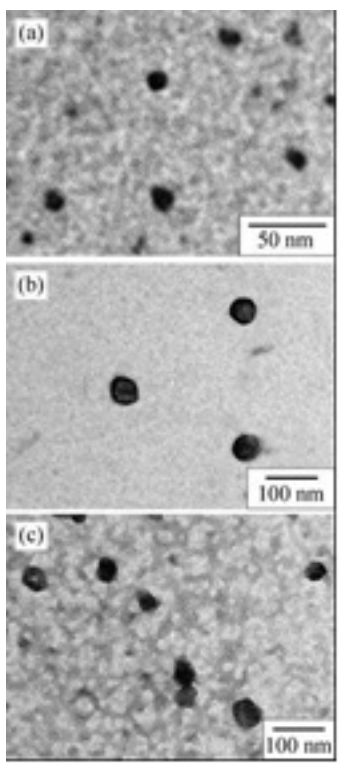

Figure 8: TEM images for (a) $A E A / E M_{27}$, (b) $A E A / E M_{53}$, and (c) $A E A / E M_{106}$ micelles.

\section{Conclusions}

Cationic diblock copolymers, $\mathrm{EM}_{m}$ with different cationic PMAPTAC block lengths and anionic triblock copolymer, AEA were prepared via RAFT radical polymerization in water using a PEG-based mono- and bifunctional CTA. The oppositely charged $\mathrm{EM}_{m}$ and AEA were mixed in aqueous solutions with stoichiometrically charge neutralized to form PIC micelles. The charge neutralization of the PIC micelles was confirmed with $\zeta$-potential. The cationic PMAPTAC block in $\mathrm{EM}_{m}$ and anionic PAMPS block in AEA formed segregated PIC core, which was confirmed with restricted motion of the ionic blocks by ${ }^{1} \mathrm{H}$ NMR. The PEG blocks formed linear and looped shell chains surrounding the PIC segregated core to form water-soluble PIC flower micelles. Light scattering and TEM data supported formation of the individual PIC flower micelles. The oppositely charged block copolymer combination with similar ionic block chain lengths formed the PIC micelle with maximum $R_{\mathrm{h}}$ and $N_{\mathrm{agg}}$. It is expected that the PIC flower micelles can be applied for the carrier of the charged drugs, because the PIC flower micelles can incorporate charged guest molecules in the PIC core. When the concentration of the PIC flower micelles increase above more than $1 \mathrm{~g} / \mathrm{L}$, the AEA and $\mathrm{EM}_{m}$ may form network structure to be gel. Currently, polymer gel formation at high polymer concentrations is studied in our laboratory.

\section{Acknowledgements}

This work was financially supported by a Grant-in-Aid for Scientific Research (25288101) from the Japan Society for the Promotion of Science (JSPS), and the Cooperative Research Program "Network Joint Research Center for Materials and Devices".

\section{References}

1. Nuopponen M, Kalliomäki K, Aseyev V, Tenhu H (2008) Spontaneous and thermally induced self-organization of A-B-A stereoblock polymers of $\mathrm{N}$-isopropylacrylamide in aqueous solutions. Macromolecules 41: 4881-4886.

2. Kadam VS, Nicol E, Gaillard C (2012) Synthesis of flower-like poly (ethylene oxide) based macromolecular architectures by photo-cross-linking of block copolymers self-assemblies. Macromolecules 45: 410-419.

3. de Graaf AJ, Boere KWM, Kemmink J, Fokkink RG, van Nostrum CF, et al., (2011) Looped structure of flowerlike micelles revealed by ${ }^{1} \mathrm{H}$ NMR relaxometry and light scattering. Langmuir 27: 9843-9848.

4. Lee W, Chang J, Ju S (2010) Hydrogen-bond structure at the interfaces between water/poly (methyl methacrylate), water/poly(methacrylic acid), and water/poly(2-aminoethylmethacrylamide). Langmuir 26: 12640-12647.

5. Foreman MB, Coffman JP, Murcia MJ, Cesana S, Jordan R, et al., (2003) Gelation of amphiphilic lipopolymers at the air-water interface: 2D analogue to $3 \mathrm{D}$ gelation of colloidal systems with grafted polymer chains. Langmuir 19: 326-332.

6. Xu R, Winnik MA, Riess G, Chu B, Croucher MD (1992) Micellization of polystyrene-poly(ethylene oxide) block copolymers in water. 5 . A test of the star and mean-field models. Macromolecules 25: 644-652.

7. Kabanov VA, Zezin AB, Kasaikin VA, Zakharova JA, Litmanovich EA, et al. (2003) Self-assembly of ionic amphiphiles on polyelectrolyte chains. Polymer International 52: 1566-1572.

8. Santis SDA, Ladogana RD, Diociaiuti M, Masci G (2010) Pegylated and thermosensitive polyion complex micelles by self-assembly of two oppositely and permanently charged diblock copolymers. Macromolecules 43: 1992-2001.

9. Harada A, Kataoka K (1995) Formation of polyion complex micelles in an aqueous milieu from a pair of oppositely-charged block copolymers with poly (ethylene glycol) segments. Macromolecules 28: 5294-5299.

10. Harada A, Kataoka K (1999) Novel polyion complex micelles entrapping enzyme molecules in the core. 2. Characterization of the micelles prepare at nonstoichiometric mixing ratios. Langmuir 15: 4208-4212.

11. Harada A, Kataoka K (2003) Effect of charged segment length on physicochemical properties of core-shell type polyion complex micelles from block ionomers. Macromolecules 36: 4995-5001.

12. Lokitz BS, Convertine AJ, Ezell RG, Heidenreich A, Li Y, et al., (2006) Responsive nanoassemblies via interpolyelectrolyte complexation of amphiphilic block copolymer micelles. Macromolecules 39: 8594-8602. 
Citation: Yokoyama Y, Enomoto R, Yusa S (2014) Polyion Complex (PIC) Flower-shaped Nano-micelles formed from Anionic Triblock and Cationic Diblock Copolymers. J Nanotechnol Nanomed Nanobiotechnol 1: 001.

13. Li Y, Lokitz BS, McComick CL (2006) Thermally responsive vesicles and their structural "locking" through polyelectrolyte complex formation. Angew Chem Int Ed 118: 5924-5927.

14. Xu X, Smith AE, Kirkland SE, McComick CL (2008) Aqueous RAFT synthesis of $\mathrm{pH}$-responsive triblock copolymer mPEO-PAPMA-PDPAEMA and formation of shell cross-linked micelles. Macromolecules 41: 8429-8435.

15. Yusa S, Yokoyama Y, Morishima Y (2009) Synthesis of oppositely charged block copolymers of poly (ethylene glycol) via reversible addition-fragmentation chain transfer radical polymerization and characterization of their polyion complex micelles in water. Macromolecules 42: 376-383.

16. Mitsukami Y, Donovan MS, Lowe AB, McCormick CL (2001) Water-soluble polymers. 81. Direct synthesis of hydrophilic styrenic-based homopolymers and block copolymers in aqueous solution via RAFT. Macromolecules 34 2248-2256.

17. Achilleos M, Legge TM, Perrier S, Patrickios CS (2008) Poly (ethylene glycol)-based amphiphilic model conetworks: Synthesis by RAFT polymerization and characterization. J Polym Sci Part A: Polym Chem 46: 7556-7565.

18. Jakes J (1995) Regularized Positive Exponential Sum (REPES) program - A way of inverting Laplace transform data obtained by dynamic light scattering. Collect Czech Chem Commun 60: 1781-1797.

19. Brown W, Nicolai T, Hvidt S, Stepanek P (1990) Relaxation time distributions of entangled polymer solutions from dynamic light scattering and dynamic mechanical measurements. Macromolecules 23: 357-359.

20. Stockmayer WH, Schmidt M (1982) Effects of polydispersity, branching and chain stiffness on quasielastic light scattering. Pure Appl Chem 54: 407-414.
21. Phillies GDJ (1990) Quasielastic light scattering. Anal Chem 62: 1049-1057.

22. Ali SI, Heuts JPA, van Herk AM (2010) Controlled synthesis of polymeric nanocapsules by RAFT-based vesicle templating. Langmuir 26: 7848-7858.

23. Donovan MS, Lowe AB, Sumerlin BS, McCormik CL (2002) RAFT polymerization of $\mathrm{N}, \mathrm{N}$-dimethylacrylamide utilizing novel chain transfer agents tailored for high reinitiation efficiency and structural control. Macromolecules 35: 4123-4132.

24. Yusa S, Fukuda K, Yamamoto T, Ishihara K, Morishima Y (2005) Synthesis of well-defined amphiphilic block copolymers having phospholipid polymer sequences as a novel biocompatible polymer micelle reagent. Biomacromolecules 6: 663-670.

25. Xu R, Winnik MA, Hallett FR, Riess G, Croucher MD (1991) Light-scattering study of the association behavior of styrene-ethylene oxide block copolymers in aqueous solution. Macromolecules 24: 87-93.

26. Huber K, Bantle S, Lutz P, Burchard W (1985) Hydrodynamic and thermodynamic behavior of short-chain polystyrene in toluene and cyclohexane at $34.5^{\circ} \mathrm{C}$. Macromolecules 18: 1461-1467.

27. Akcasu AZ, Han CC (1979) Molecular weight and temperature dependence of polymer dimensions in solution. Macromolecules 12: 276-280.

28. Konishi T, Yoshizaki T, Yamakawa H (1991) On the "universal constants" $\rho$ and $\Phi$. of flexible polymers. Macromolecules 24: 5614-5622.

29. Zhang M, Xue YN, Liu M, Zhuo RX, Huang SW (2010) Biocleavable polycationic micelles as highly efficient gene delivery vectors. Nanoscale Res Lett 5: 1804-1811. 